\title{
A Revisited Half-Quadratic Approach for Simultaneous Robust Fitting of Multiple Curves
}

\author{
Jean-Philippe Tarel $^{1}$, Pierre Charbonnier ${ }^{2}$, and Sio-Song Ieng ${ }^{1}$ \\ 1 ESE, Laboratoire Central des Ponts et Chaussées, \\ 58 Bd Lefebvre, 75015 Paris, France \\ Tarel@lcpc.fr, Sio-Song. Ieng@lcpc.fr, \\ http://perso.lcpc.fr/tarel.jean-philippe/ \\ 2 ERA 27 LCPC, Laboratoire des Ponts et Chaussées, \\ 11 rue Jean Mentelin, B.P. 9, 67035 Strasbourg, France \\ Pierre.Charbonnier@equipement.gouv.fr
}

\begin{abstract}
In this paper, we address the problem of robustly recovering several instances of a curve model from a single noisy data set with outliers. Using M-estimators revisited in a Lagrangian formalism, we derive an algorithm that we call Simultaneous Multiple Robust Fitting (SMRF), which extends the classical Iterative Reweighted Least Squares algorithm (IRLS). Compared to the IRLS, it features an extra probability ratio, which is classical in clustering algorithms, in the expression of the weights. Potential numerical issues are tackled by banning zero probabilities in the computation of the weights and by introducing a Gaussian prior on curves coefficients. Applications to camera calibration and lane-markings tracking show the effectiveness of the SMRF algorithm, which outperforms classical Gaussian mixture model algorithms in the presence of outliers.
\end{abstract}

Key words: Image Analysis, Statistical Approach, Robust Fitting, Multiple Fitting, Image Grouping and Segmentation

\section{Introduction}

In this paper, we propose a method for robustly recovering several instances of a curve model from a single noisy data set with severe perturbations (outliers). It is based on an extension of the work reported in [1], in which M-estimators are revisited in an Lagrangian formalism, leading to a new derivation and convergence proof of the well-known Iterative Reweighted Least Squares (IRLS) algorithm. Following the same approach based on the Lagrangian framework, we derive, in a natural way, a deterministic, alternate minimization algorithm for multiple regression, called Simultaneous Multiple Robust Fitting (SMRF) algorithm. The SMRF can be seen as an extension of the IRLS algorithm, in which an extra probability ratio, which is classical in clustering algorithms, appears in the expression of the weights. To tackle potential numerical issues, zero probabilities are banned in the computation of the weights and a Gaussian prior on the curves coefficients is introduced. Such a prior is, moreover, well-suited 
to sequential image processing and provides control on the curves. Applications to camera calibration and lane-markings tracking illustrate the effectiveness of the SMRF algorithm. In particular, it outperforms classical Gaussian mixture model algorithms in the presence of outliers.

The paper is organized as follows. In Sec. 2, we present the robust multiple curves estimation problem and introduce our algorithmic strategy. The resulting algorithm is given in Sec. 3. Technical details on its derivation and convergence proof are given in the Appendix. In Sec. 4, connections are made with other approaches in the domain. Finally, we apply the algorithm to road tracking and to camera calibration, in Sec. 5 .

\section{Multiple robust Maximum Likelihood Estimation (MLE)}

In this section, we model the problem of simultaneously fitting $m$ curves in a robust way. Each individual curve is explicitly described by a vector parameter $\tilde{A}_{j}, 1 \leq j \leq m$. The observations, $y$, are given by a linear generative model:

$$
y=X(x)^{t} \tilde{A}_{j}+b
$$

where $(x, y)$ are the image coordinates of a data point, $\tilde{A}_{j}=\left(\tilde{a}_{i j}\right)_{0 \leq i \leq d}$ is the vector of curve parameters and $X(x)=\left(f_{i}(x)\right)_{0 \leq i \leq d}$ is the vector of basis functions at the image coordinate $x$, which will be denoted as $X$ for the sake of simplicity. These vectors are of size $d+1$. Example of basis functions will be given in Sec. 5.2. Note that we consider the fixed design case, i.e. in (1), $x$ is assumed non-random. In that case, it is shown that certain M-estimators attain the maximum possible breakdown point of approximately $50 \%$ [2]. In all that follows, the measurement noise $b$ is assumed independent and identically distributed (iid) and centered. In order to render the estimates robust to nonGaussian noise (outliers), we formulate the noise distribution as:

$$
p_{s}(b) \propto \frac{1}{s} e^{-\frac{1}{2} \phi\left(\left(\frac{b}{s}\right)^{2}\right)}
$$

where $\propto$ denotes the equality up to a factor, and $s$ is the scale of the pdf. As stated in [3], the role of $\phi$ is to saturate the error in case of a large noise $|b|=$ $\left|X^{t} \tilde{A}_{j}-y\right|$, and thus to lower the importance of outliers. The scale parameter, $s$, controls the distance from which noisy measurements have a good chance of being considered as outliers. The algorithm derivation is performed using the half-quadratic approach $[4,5]$ revisited using classical optimization tools, namely Lagrange duality [1]. The potential function $\phi(t)$ must fulfill the following hypotheses:

- H0: defined and continuous on $[0,+\infty[$ as well as its first and second derivatives,

- H1: $\phi^{\prime}(t)>0$ (thus $\phi$ is increasing),

- H2: $\phi^{\prime \prime}(t)<0$ (thus $\phi$ is concave). 
Note that these assumptions are very different from those used in [3], where the convergence proof required that the potential function $\rho(b)=\phi\left(b^{2}\right)$ be convex. In the present case, the concavity and monotonicity requirements imply that $\phi^{\prime}(t)$ is bounded, but $\phi\left(b^{2}\right)$ is not necessarily convex w.r.t. $b$.

Our goal is to simultaneously estimate the $m$ curve parameter vectors $A_{j=1, \cdots, m}$ from the whole set of $n$ data points $\left(x_{i}, y_{i}\right), i=1, \cdots, n$. The probability of a measurement point $\left(x_{i}, y_{i}\right)$, given the $m$ curves is the sum of the probabilities over each curve:

$$
p_{i}\left(\left(x_{i}, y_{i}\right) \mid A_{j=1, \cdots, m}\right) \propto \frac{1}{s} \sum_{j=1}^{j=m} e^{-\frac{1}{2} \phi\left(\left(\frac{X_{i}^{t} A_{j}-y_{i}}{s}\right)^{2}\right)} .
$$

Concatenating all curve parameters into a single vector $A=\left(A_{j}\right), j=1, \cdots, m$ of size $m(d+1)$, we can write the probability of the whole set of points as the product of the individual probabilities:

$$
p\left(\left(x_{i}, y_{i}\right)_{i=1, \cdots, n} \mid A\right) \propto \frac{1}{s^{n}} \prod_{i=1}^{i=n} \sum_{j=1}^{j=m} e^{-\frac{1}{2} \phi\left(\left(\frac{X_{i}^{t} A_{j}-y_{i}}{s}\right)^{2}\right)}
$$

Maximizing the likelihood $p\left(\left(x_{i}, y_{i}\right)_{i=1, \cdots, n} \mid A\right)$ is equivalent to minimizing the negative of its logarithm:

$$
e_{M L E}(A)=\sum_{i=1}^{i=n}-\ln \left(\sum_{j=1}^{j=m} e^{-\frac{1}{2} \phi\left(\left(\frac{X_{i}^{t} A_{j}-y_{i}}{s}\right)^{2}\right)}\right)+n \ln (s)
$$

Using the same trick as the one described in [1] for robust fitting of a single curve, we introduce the auxiliary variables $w_{i j}=\left(\frac{X_{i}^{t} A_{j}-y_{i}}{s}\right)^{2}$, as explained in the Appendix. We then rewrite the value $e_{M L E}(A)$ as the value achieved at the unique saddle point of the following Lagrange function:

$$
L_{R}=\sum_{i=1}^{i=n} \sum_{j=1}^{j=m} \frac{1}{2} \lambda_{i j}\left(w_{i j}-\left(\frac{X_{i}^{t} A_{j}-y_{i}}{s}\right)^{2}\right)+\sum_{i=1}^{i=n} \ln \left(\sum_{j=1}^{j=m} e^{-\frac{1}{2} \phi\left(w_{i j}\right)}\right)-n \ln (s)
$$

Then, the algorithm obtained by alternated minimizations of the dual function w.r.t. $\lambda_{i j}$ and $A$ is globally convergent, towards a local minimum of $e_{M L E}(A)$, as shown in the Appendix.

\section{Simultaneous Multiple Robust Fitting Algorithm}

As detailed in the Appendix, minimizing (5) leads to alternate between the three sets of equations:

$$
w_{i j}=\left(\frac{X_{i}^{t} A_{j}-y_{i}}{s}\right)^{2}, 1 \leq i \leq n, 1 \leq j \leq m,
$$




$$
\begin{aligned}
\lambda_{i j}= & \frac{e^{-\frac{1}{2} \phi\left(w_{i j}\right)}}{\sum_{k=1}^{k=m} e^{-\frac{1}{2} \phi\left(w_{i k}\right)}} \phi^{\prime}\left(w_{i j}\right), 1 \leq i \leq n, 1 \leq j \leq m, \\
& \left(\sum_{i=1}^{i=n} \lambda_{i j} X_{i} X_{i}^{t}\right) A_{j}=\sum_{i=1}^{i=n} \lambda_{i j} y_{i} X_{i}, 1 \leq j \leq m
\end{aligned}
$$

In practice, some care must be taken, to avoid numerical problems and singularities. First, it is important that the denominator in (7) be numerically non-zero, which might occur for a data point located far from all curves. Zero probabilities are banned by adding a small value $\epsilon$ (equal to the machine precision) to the exponential in the probability $p_{i}$ of a measurement point. As a consequence, when a point with index $i$ is far from all curves, $\phi^{\prime}\left(w_{i j}\right)$ is weighted by a constant factor, $1 / m$, in (7).

Second, the linear system in (8) can be singular. To avoid this, it is necessary to enforce a Gaussian prior on the whole curves parameters with bias $A^{p r}$ and covariance matrix $C^{p r}$. Note that the reason for introducing such a prior is not purely technical: it is indeed a very simple and useful way of taking into account application-specific a priori knowledge, as shown in Sec. 5.3 and 5.4. As a default prior, we suppose that the bias is zero, i.e $A^{p r}=0$, and that the inverse covariance matrix is block diagonal where each diagonal block equals:

$$
C^{p r-1}=r \int_{-1}^{1} X(x) X(x)^{t} d x
$$

assuming that $[-1,1]$ is the range where $x$ varies. The integral is the inverse covariance matrix of the curve fitting estimator under a Gaussian noise assumption which can be used for approximately modeling the truncation errors due to image sampling. The default prior also accounts for possible correlations between basis functions, which can be helpful when using non-orthogonal bases. The regularization term $\left(A-A^{p r}\right)^{t} C^{p r-1}\left(A-A^{p r}\right)$ is added to (4) and (5). Therefore, the parameter $r$ controls the balance between the data fidelity term and the prior.

Finally, the Simultaneous Multiple Robust Fitting algorithm (SMRF) is:

1. Initialize the number of curves $m$, the vector $A^{0}=\left(A_{j}^{0}\right), 1 \leq j \leq m$, which gathers all curves parameters and set the iteration index to $k=1$.

2. For all indexes $i, 1 \leq i \leq n$, and $j, 1 \leq j \leq m$, compute the auxiliary variables $w_{i j}^{k}=\left(\frac{X_{i}^{t} A_{j}^{k-1}-y_{i}}{s}\right)^{2}$ and the weights $\lambda_{i j}^{k}=\frac{\epsilon+e^{-\frac{1}{2} \phi\left(w_{i j}^{k}\right)}}{m \epsilon+\sum_{j=1}^{j=m} e^{-\frac{1}{2} \phi\left(w_{i j}^{k}\right)}} \phi^{\prime}\left(w_{i j}^{k}\right)$.

3. Solve the linear system:

$$
\left[D+C^{p r-1}\right] A^{k}=\left[\begin{array}{c}
\sum_{i=1}^{i=n} \lambda_{i 1}^{k} y_{i} X_{i} \\
\vdots \\
\sum_{i=1}^{i=n} \lambda_{i m}^{k} y_{i} X_{i}
\end{array}\right]+C^{p r-1} A^{p r}
$$

4. If $\left\|A^{k}-A^{k-1}\right\|>\epsilon^{\prime}$, increment $k$, and go to 2 , else the solution is $A=A^{k}$. 
In the above algorithm, $D$ is the block-diagonal matrix whose $m$ diagonal blocks are the matrices $\sum_{i=1}^{i=n} \lambda_{i j}^{k} X_{i} X_{i}^{t}$ of size $(d+1) \times(d+1)$, with $1 \leq j \leq m$. The prior covariance matrix $C^{p r}$ is of size $m(d+1) \times m(d+1)$. The prior bias $A^{p r}$ is a vector of size $m(d+1)$, as well as $A$ and $A^{k}$. The complexity is $O(n m)$ for the step 2, and $O\left(m^{2}(d+1)^{2}\right)$ for the step 3 of the algorithm.

\section{Connections with other approaches}

The proposed algorithm has important connections with previous works in the field of regression and clustering and we would like to highlight a few of them.

In the single curve case, $m=1$, the SMRF algorithm is reduced to the socalled Iterative Reweighted Least Squares extensively used in M-estimators [3], half-quadratic theory $[5,4]$, and others. The SMRF and IRLS algorithms share very similar structures and it is important to notice that the main difference lies within the Lagrange multipliers $\lambda_{i j}$, see (7). Compared to the IRLS, the $\lambda_{i j}$ are just weighted by an extra probability ratio, which is classical in clustering algorithms.

To make the connection with clustering clearer, let us substitute $Y=A_{j}+b$ to the generative model (1), where $Y$ and $A_{j}$ are vectors of same size and respectively denote a data points and a cluster centroid. The derivation described in Sec. 3 is still valid and the obtained algorithm turns to be a clustering algorithm with $m$ clusters, each cluster being represented by a vector, its centroid. The probability distribution of a cluster around its centroid is directly specified by the function $\phi$. The obtained algorithm is thus able of modeling the $Y_{i}$ 's by a mixture of pdfs which are not necessarily Gaussian. The mixture problem is usually solved by the well-known Expectation-Minimization (EM) approach [6]. In the non-Gaussian case, the minimization (M) step implements robust estimation, which is an iterative process in itself. Hence, the resulting EM algorithm involves two nested loops, while the proposed algorithm features only one. An alternative to the EM approach is the Generalized EM (GEM) approach which consists in performing an approximate M-step: typically, only one iteration rather than the full minimization. The resulting algorithm in the robust case is identical to the one we derived within the Lagrangian framework (apart from the regularization of the singular cases). In our formalism however, no approximation is made in the derivation of the algorithm, in contrast with the GEM approach.

We also found that the SMRF algorithm is very close to an earlier work in the context of clustering [7]. However, to our knowledge, the latter was just introduced as an extra ad-hoc weighting over M-estimators without statistical interpretation and, moreover, singular configurations were not dealt with.

The SMRF algorithm is subject to the initialization problem since it only converges towards a local minimum. To tackle this problem, the Graduated Non Convexity approach (GNC) [8] is used to improve the chances of converging towards the global minimum. Details are given in Sec. 5.4. The SMRF can be also used as a fitting process within the RANSAC [9] approach to improve the convergence towards the global minimum. 


\section{Experimental Results}

The proposed approach being based on a linear generative model, many applications could potentially be addressed using the SMRF algorithm. In this paper, we focus on two specific applications, namely simultaneous lane-markings tracking and camera calibration from a regular lattice of lines with geometric distortions. See [13] for more detail information.

\subsection{Noise Model}

Among the suitable functions for robust estimation, we use a simple parametric family of probability distribution functions, that was introduced in [1] under the name of smooth exponential family $(\mathrm{SEF}), S_{\alpha, s}$ :

$$
S_{\alpha, s}(b) \propto \frac{1}{s} e^{-\frac{1}{2} \phi_{\alpha}\left(\left(\frac{b}{s}\right)^{2}\right)}
$$

where, with $t=\left(\frac{b}{s}\right)^{2}, \phi_{\alpha}(t)=\frac{1}{\alpha}\left((1+t)^{\alpha}-1\right)$.

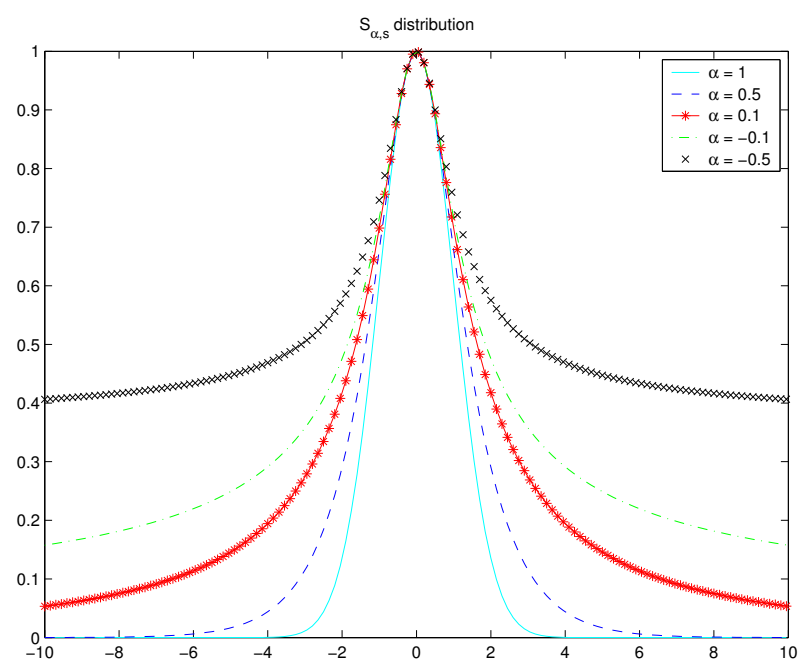

Fig. 1. Noise models in the SEF $S_{\alpha, s}$. Notice how the tails become heavier as $\alpha$ decreases.

These laws are shown in Figure 1 for different values of $\alpha$. The smaller the value of $\alpha$, the higher the probability of observing large, not to say very large, errors (outliers). This parameter allows a continuous transition between wellknown statistical laws such as Gauss $(\alpha=1)$, smooth Laplace $\left(\alpha=\frac{1}{2}\right)$ and T-Student $(\alpha \rightarrow 0)$. This can be exploited to get better convergence of the SMRF algorithm by using the GNC approach, i.e. by progressively decreasing $\alpha$ towards 0 . 


\subsection{Road Shape Model}

The road shape features $(x, y)$ are given by the lane-marking centers extracted using the local feature extractor described in [10]. An example of extraction is shown in Figure 6(b). In practice, we model road lane markings by polynomials $y=\sum_{i=0}^{d} a_{i} x^{i}$. Moreover, in the flat world approximation, the image of a polynomial on the road under perspective projection is a hyperbolic polynomial with equation $y=c_{0} x+c_{1}+\sum_{i=2}^{d} \frac{c_{i}}{\left(x-x_{h}\right)^{2}}$, where $c_{i}$ is linearly related to $a_{i}$. Therefore, the hyperbolic polynomial model is well suited to the case of road scene analysis. To avoid numerical problems, a whitening of the data is performed by scaling the image in a $[-1,1] \times[-1,1]$ box for polynomial curves and in a $[0,1] \times[-1,1]$ box for hyperbolic polynomials, prior to the fitting.

\subsection{Geometric Priors}

As noticed in Sec. 3, the use of a Gaussian prior allows introducing useful application-specific knowledge. For example, using (9) for the diagonal blocks of the inverse prior covariance matrix, we take into account perturbations due to image sampling.

Tuning the diagonal elements of $C^{p r}$ provides control on the curve degree. For polynomials, the diagonal components of the covariance matrix correspond to monomials of different degrees. The components of degree higher than one are thus set to smaller values than those of degree zero and one.

Geometric smooth constraints between curves can be enforced by using also non-zero off-diagonal blocks. In particular, it is a soft way of maintaining parallelism between curves. As an illustration, considering two lines $y=a_{0}+a_{1} x$ and $y=a_{0}^{\prime}+a_{1}^{\prime} x$, the prior covariance matrix is obtained by rewriting $\left(a_{1}-a_{1}^{\prime}\right)^{2}$ in matrix notations:

$$
\left[\begin{array}{l}
a_{0} \\
a_{1} \\
a_{0}^{\prime} \\
a_{1}^{\prime}
\end{array}\right]^{t}\left[\begin{array}{cccc}
0 & 0 & 0 & 0 \\
0 & 1 & 0 & -1 \\
0 & 0 & 0 & 0 \\
0 & -1 & 0 & 1
\end{array}\right]\left[\begin{array}{c}
a_{0} \\
a_{1} \\
a_{0}^{\prime} \\
a_{1}^{\prime}
\end{array}\right]
$$

The above matrix, multiplied by an overall factor can be used as an inverse prior covariance $C^{p r-1}$. The factor controls the balance between the data fidelity term and the other priors. Other kinds of geometric smooth constraints can be handled in a similar way, such as intersection at a given point, or symmetric orientations. These geometric priors can be combined by adding the associated regularization term $\left(A-A^{p r}\right)^{t} C^{p r-1}\left(A-A^{p r}\right)$ to (4) and (5).

\subsection{Lane-Markings Tracking}

We shall now describe the application of the SMRF algorithm to the problem of tracking lane markings.

In addition to the previous section, another interesting feature of using a Gaussian prior is that the SMRF is naturally suitable for being included in a 
Kalman filtering. However, this raises the question of the definition of the posterior covariance matrix of the estimate. Under the Gaussian noise assumption, the estimate of the posterior covariance matrix is well-known for each curve: $C_{j}=s^{2}\left(\sum_{i=1}^{i=n} X_{i} X_{i}^{t}\right)^{-1}$. Unfortunately, in the context of robust estimation, the estimation of $C_{j}$ for each curve $A_{j}$ is a difficult issue and only approximate matrices are available. In [10], several approximates were compared. The underlying assumption for defining all these approximates is that the noise is independent. However, we found out that in practice, the noise is correlated from one image line to another. Therefore, all these approximates can be improved by introducing an had-hoc correction factor which accounts for data noise correlations in the inverse covariance matrix. We experimentally found that the following factor is appropriate, for each curve $j$ :

$$
1-\frac{\sum_{i=1}^{i=n-1} \sqrt{\lambda_{i j} w_{i j} \lambda_{i+1, j} w_{i+1, j}}}{\sum_{i=1}^{i=n} \lambda_{i j} w_{i j}}
$$

The approximate posterior covariance matrix for the whole set of curve parameters, $A$, is simply built as a block-diagonal matrix made of the individual posterior covariance matrices for each curve, $C_{j}$. This temporal prior can be easily combined with geometric priors for tracking parallel curves, for instance.

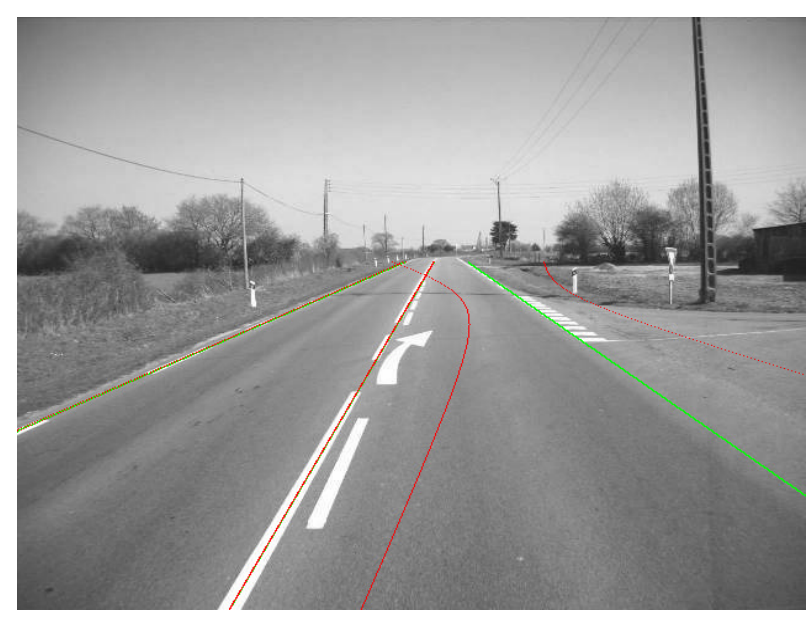

Fig. 2. Detected lane-markings (in green) and uncertainty about curve position (in red).

Figure 2 shows the three curves simultaneously fitted on the lane-marking centers (in green) and the associated uncertainty curves of the horizontal position of each fitted curve $\left( \pm \sqrt{X(x)^{t} C_{j}^{-1} X(x)}\right.$, in red). Notice that the uncertainty on the right sparse lane-marking is higher than for the continuous one on the center. Moreover, the higher the distance to the camera, the higher the uncertainty, since 
the curve gets closer to possible outliers. In all these experiments, and following, the parameters used for the noise model are $\alpha=0.1$ and $s=4$.

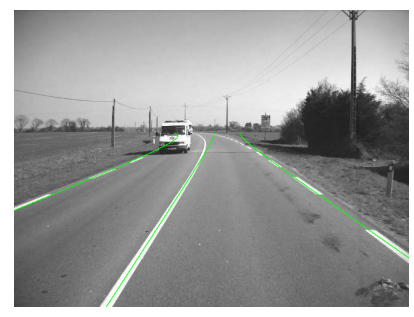

(a)

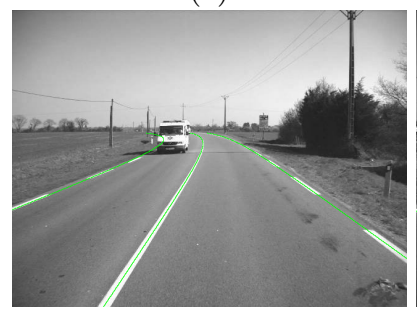

(c)

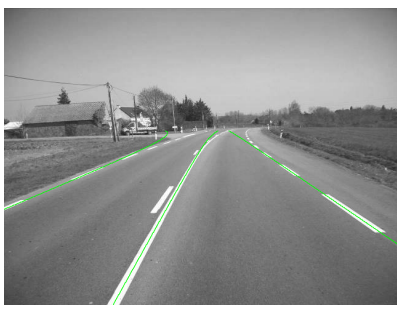

(b)

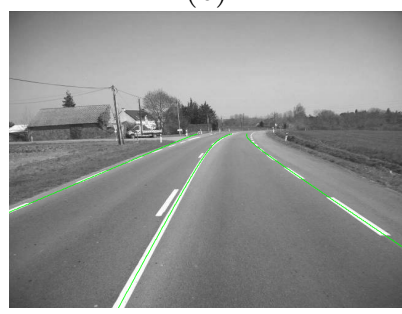

(d)

Fig. 3. Two images extracted from a sequence of 240 images processed with, on (a)(b), separate Kalman filters and, on (c)(d), simultaneous Kalman filter. The three detected lane-markings of degree two are in green.

For the tracking itself, we experimented both separate Kalman filters on individual curves, and a simultaneous Kalman filter. The former can be seen as a particular case of the latter, in which the inverse prior covariance matrix $C^{p r}$ is block-diagonal so the linear system of size $m(d+1)$ in the SMRF algorithm can be decomposed as $m$ linear independent systems of size $d+1$. Figure 3 compares the results obtained with separate and simultaneous Kalman filters. Notice how the parallelism between curves is better preserved within the simultaneous Kalman filter, thanks to an adequate choice of the off-diagonal blocks of $C^{p r}$.

Figure 4 illustrates the ability of the SMRF-based Kalman filter to fit and track several curves in an image sequence. In that case, three lane-markings are simultaneously fitted and correctly tracked, even though the vehicle performs several lane changes during the 150-image sequence. Notice that, while Kalman filtering can incorporate a dynamic model of the vehicle, we only used a static model in these experiments, since only the images were available. We observed that it is better to initialize the SMRF algorithm with the parameters resulting from the fitting on the previous image, rather than with the filtered parameters: filtering indeed introduces a delay in the case of fast displacements or variations of the tracked curves.

Moreover, we obtained interesting results on difficult road sequences. For instance, Figure 5 shows a short sequence of poor quality images, due to rain. 


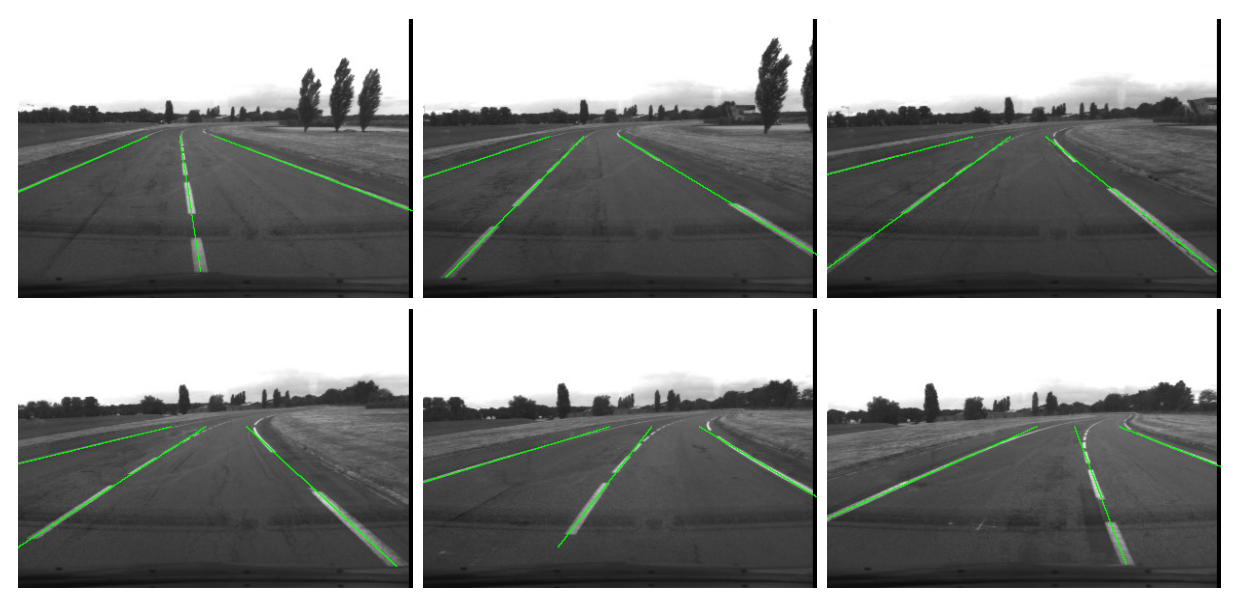

Fig. 4. Six of a 150-image sequence, featuring lane changes. Green lines show the three fitted lane-markings centers.

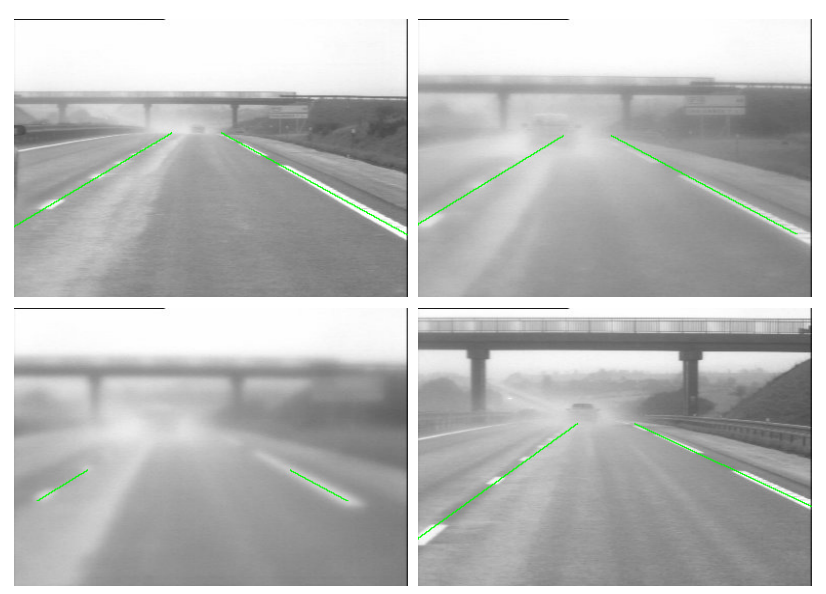

Fig. 5. Fitting in adverse conditions: in this excerpt, the left lane-marking is mostly hidden on two successive images.

The left lane-marking is mostly hidden on two consecutive images. Thanks to the simultaneous Kalman filter, the SMRF algorithm is able to interpolate correctly the hidden lane-marking.

\subsection{Camera Calibration}

We now present another application of the SMRF algorithm, in the context of camera calibration. The goal is to estimate accurately the position and orientation of the camera with respect to the road and its intrinsic parameters. A calibration setup made of two sets of perpendicular lines painted on the road is 


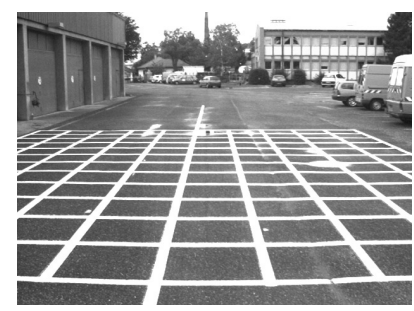

(a)

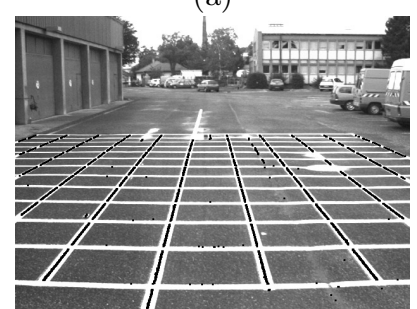

(d)

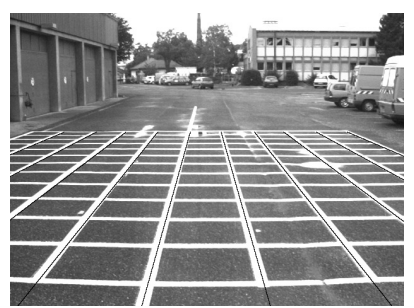

(b)

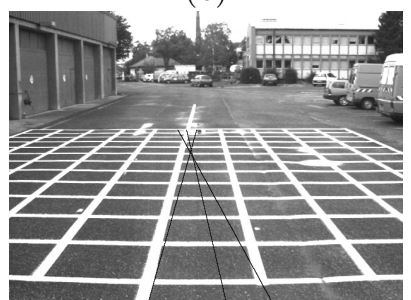

(e)

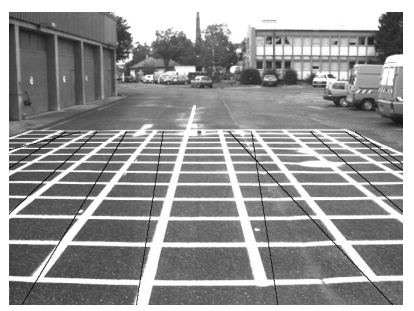

(c)

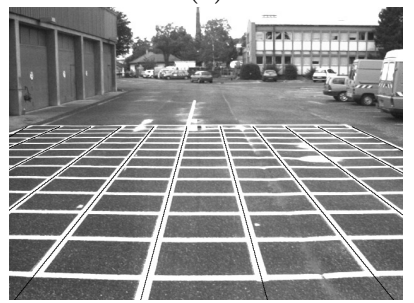

(f)

Fig. 6. (a) Original image of the calibration grid. (d) Extracted lane-marking centers (outliers are due to puddles). (b) 10 initial lines for the fitting on the vertical markings. (e) Fitted lines on the vertical markings under Gaussian noise assumption. (c) 12 initial lines for the fitting on the vertical markings. (f) The robust fitting yields 11 different correct lines.

thus observed by a camera mounted on a vehicle, as shown in Figure 6(a). The SMRF algorithm can be used to provide accurate data to the calibration algorithm by estimating the grid intersections. Even though the markings are clearly visible in the image, some of them are quite short, and there are outliers due to the presence of water puddles. Figure $6(\mathrm{~d})$ shows the extracted lane-marking centers. When a Gaussian mixture model is used, the obtained fit is severely troubled by the outliers, as displayed in Figure 6(e), even though the curves are initialized very close to the expected solution, see Figure 6(b).

On the contrary, with the same extracted lane-marking centers, the SMRF algorithm, with noise model parameters $\alpha=0.1$ and $s=4$, leads to nice results, as shown in Figure $6(\mathrm{f})$ for the vertical lines. 11 different lines were obtained for the vertical markings, even if the initial curves where not very close to the solution as illustrated by Figure 6(c).

\section{Conclusion}

In the continuing quest for achieving robustness in detection and tracking curves in images, this paper makes two contributions. The first one is the derivation, in a MLE approach and using Kuhn and Tucker's classical theorem, of the so-called SMRF algorithm. This algorithm extends mixture model algorithm, such as the one derived using EM, to robust curve fitting. It is also an extended version 
of the IRLS, in which the weights incorporate an extra probability ratio. The second contribution is the regularization of the SMRF algorithm by introducing Gaussian priors on curve parameters and the handling of potential numerical issues by banning zero probabilities in the computation of weights. ¿From our experiments, banning zero probabilities seems to have important positive consequences in pushing the curves to spread out all the data, and thus in providing improved robustness to the initialization, as shown in the context of camera calibration. The introduction of the Gaussian prior is also beneficial in particular in the context of image sequence processing, as illustrated with an application of simultaneous lane-markings tracking on-board a vehicle in adverse conditions. The approach being based on a linear generative model, it is quite generic and we believe that it can be used with benefits in many other fields of computer vision, such as clustering or appearance modeling, registration, parametric region fitting, as illustrated in $[11,12,13]$.

\section{Appendix}

We shall first rewrite the value $-e_{M L E}(A)$ for any given $A=\left(A_{j}\right), j=1, \cdots, m$ as the value achieved at the minimum of a convex problem under convex constraints. This is obtained by introducing the auxiliary variables $w_{i j}=$ $\left(\frac{X_{i}^{t} A_{j}-y_{i}}{s}\right)^{2}$. This apparent complication is in fact valuable since it allows introducing Lagrange multipliers, and thus to decompose the original problem in simpler problems. The value $-e_{M L E}(A)$ can be seen as the minimum value, w.r.t. $W=\left(w_{i j}\right)_{1 \leq i \leq n, 1 \leq j \leq m}$, of:

$$
E(A, W)=\sum_{i=1}^{i=n} \ln \left(\sum_{j=1}^{j=m} e^{-\frac{1}{2} \phi\left(w_{i j}\right)}\right)
$$

subject to $n m$ constraints $h_{i j}(A, W)=w_{i j}-\left(\frac{X_{i}^{t} A_{j}-y_{i}}{s}\right)^{2} \leq 0$. This is proved by showing that the bound on each $w_{i j}$ is always achieved. Indeed $E(A, W)$ is decreasing w.r.t. each $w_{i j}$, since its first derivative:

$$
\frac{\partial E}{\partial w_{i j}}=-\frac{1}{2} \frac{e^{-\frac{1}{2} \phi\left(w_{i j}\right)}}{\sum_{k=1}^{k=m} e^{-\frac{1}{2} \phi\left(w_{i k}\right)}} \phi^{\prime}\left(w_{i j}\right)
$$

is always negative, due to (H1).

To prove the local convergence of the SMRF algorithm in Sec. 3, we now focus on the minimization of $E(A, W)$ w.r.t. $W$ only, subject to the $n m$ constraints $h_{i j}(A, W) \leq 0$, w.r.t. $W$, for any $A$. We now introduce a classical result of convex analysis [14]: the function $g(Z)=\log \left(\sum_{j=1}^{j=m} e^{z_{j}}\right)$ is convex. Due to $(\mathrm{H} 1)$ and $(\mathrm{H} 2),-\phi(w)$ is convex and decreasing. Therefore, $E(A, W)$ w.r.t. $W$ is convex as a sum of functions $g$ composed with $-\phi$, see [14]. As a consequence, the minimization of $E(A, W)$ w.r.t. $W$ is well-posed because it is a minimization of a convex function subject to convex (linear) constraints. We are thus allowed 
to apply Kuhn and Tucker's classical theorem [15]: if a solution exists, the minimization of $E(A, W)$ w.r.t. $W$ is equivalent to searching from the unique saddle point of the Lagrange function of the problem:

$$
\begin{aligned}
L_{R}(A, W, \Lambda)= & \sum_{i=1}^{i=n} \ln \left(\sum_{j=1}^{j=m} e^{-\frac{1}{2} \phi\left(w_{i j}\right)}\right) \\
& +\sum_{i=1}^{i=n} \sum_{j=1}^{m} \frac{1}{2} \lambda_{i j}\left(w_{i j}-\left(\frac{X_{i}^{t} A_{j}-y_{i}}{s}\right)^{2}\right)
\end{aligned}
$$

where $\Lambda=\left(\lambda_{i j}\right), 1 \leq i \leq n, 1 \leq j \leq m$ are Kuhn and Tucker multipliers $\left(\lambda_{i j} \geq 0\right)$. More formally, we have proved for any $A$ :

$$
-e_{M L E}(A)=\min _{W} \max _{\Lambda} L_{R}(A, W, \Lambda)
$$

Notice that the Lagrange function $L_{R}$ is quadratic w.r.t. $A$, unlike the original error $e_{M L E}$. Using the saddle point property, we can change the order of variables $W$ and $\Lambda$ in (11). We now introduce the dual function $\mathcal{E}(A, \Lambda)=$ $\min _{W} L_{R}(A, W, \Lambda)$, and rewrite the original problem as the equivalent following problem:

$$
\min _{A} e_{M L E}(A)=\min _{A, \Lambda}-\mathcal{E}(A, \Lambda)
$$

The algorithm consists in minimizing $-\mathcal{E}(A, \Lambda)$ w.r.t. $A$ and $\Lambda$ alternately. $\min _{\Lambda}-\mathcal{E}(A, \Lambda)$ leads to Kuhn and Tucker's conditions:

$$
\begin{gathered}
\lambda_{i j}=\frac{e^{-\frac{1}{2} \phi\left(w_{i j}\right)}}{\sum_{k=1}^{k=m} e^{-\frac{1}{2} \phi\left(w_{i k}\right)}} \phi^{\prime}\left(w_{i j}\right) \\
w_{i j}=\left(\frac{X_{i}^{t} A_{j}-y_{i}}{s}\right)^{2}
\end{gathered}
$$

and $\min _{A_{j}}-\mathcal{E}(A, \Lambda)$ leads to:

$$
\left(\sum_{i=1}^{i=n} \lambda_{i j} X_{i} X_{i}^{t}\right) A_{j}=\sum_{i=1}^{i=n} \lambda_{i j} y_{i} X_{i}, 1 \leq j \leq m
$$

Using classical results, see e.g. [15], $-\mathcal{E}(A, \Lambda)$ is proved to be convex w.r.t. $\Lambda$. The dual function is clearly quadratic and convex w.r.t. $A$. As a consequence, this implies that such an algorithm always strictly decreases the dual function if the current point is not a stationary point (i.e a point where the first derivatives are all zero) of the dual function [16]. The problem of stationary points is easy to solve by checking the positiveness of the Hessian matrix of $\mathcal{E}(A, \Lambda)$. If this matrix is not positive, we disturb the solution so that it converges to a local minimum. This proves that the algorithm is globally convergent, i.e, it converges toward a local minimum of $e_{M L E}(A)$ for all initial $A_{0}$ 's which are neither a maximum nor a saddle point. 


\section{References}

1. J.-P. Tarel, S.-S. Ieng, and P. Charbonnier. Using robust estimation algorithms for tracking explicit curves. In European Conference on Computer Vision (ECCV'02), volume 1, pages 492-507, Copenhagen, Danmark, 2002.

2. I. Mizera and C. Müller. Breakdown points and variation exponents of robust M-estimators in linear models. The Annals of Statistics, 27(4):1164-1177, 1999.

3. P. J. Huber. Robust Statistics. John Wiley and Sons, New York, New York, 1981.

4. D. Geman and G. Reynolds. Constrained restoration and the recovery of discontinuities. IEEE Transactions on Pattern Analysis and Machine Intelligence, 14(3):367-383, 1992.

5. P. Charbonnier, L. Blanc-Féraud, G. Aubert, and M. Barlaud. Deterministic edgepreserving regularization in computed imaging. IEEE Transactions on Image Processing, 6(2):298-311, 1997.

6. A. Dempster, N. Laird, and D. Rubin. Maximum likelihood from incomplete data via the EM algorithm. Journal of the Royal Statistical Society, Series B (Methodological), 39(1):1-38, 1977.

7. N. A. Cambell. Mixture models and atypical values. Mathematical Geology, 16(5):465-477, 1984.

8. A. Blake and A. Zisserman. Visual Reconstruction. MIT Press, Cambridge, MA, 1987.

9. R. I. Hartley and A. Zisserman. Multiple View Geometry in Computer Vision. Cambridge University Press, ISBN: 0521540518, second edition, 2004.

10. S.-S. Ieng, J.-P. Tarel, and P. Charbonnier. Evaluation of robust fitting based detection. In Proceedings of European Conference on Computer Vision (ECCV'O4), pages 341-352, Prague, Czech Republic, 2004.

11. E. Bigorgne and J.-P. Tarel. Backward segmentation and region fitting for geometrical visibility range estimation. In Proceedings of Asian Conference on Computer Vision (ACCV'07), volume II, pages 817-826, Tokyo, Japan, 2007.

12. J.-P. Tarel, S.-S. Ieng, and P. Charbonnier. Accurate and robust image alignment for road profile reconstruction. In Proceedings of IEEE International Conference on Image Processing (ICIP'07), volume V, pages 365-368, San Antonio, Texas, USA, 2007.

13. J.-P. Tarel, S.-S. Ieng, and P. Charbonnier. Robust Lane Marking Detection by the Half Quadratic Approach. Collection Etudes et Recherches des Laboratoires des Ponts et Chaussées, CR 49, LCPC, November 2007.

14. S. Boyd and L. Vandenberghe. Convex Optimization. Cambridge University Press, 2004.

15. M. Minoux. Mathematical Programming: Theory and Algorithms. Chichester: John Wiley and Sons, 1986.

16. D. G. Luenberger. Introduction to linear and nonlinear programming. Addison Wesley, 1973. 